\title{
Designing and testing of a sensor based on a magnetoresistive manganese perovskite thick film
}

\author{
LL. Balcells, R. Enrich, A. Calleja, J. Fontcuberta, and X. Obradors \\ Institut de Ciència de Materials de Barcelona, Consejo Superior de Investigaciones Científicas, Campus \\ Universitat Autònoma de Barcelona, Bellaterra 08193, Catalunya, Spain
}

In this paper we report on the growth of thick films of magnetoresistive $\mathrm{La}_{2 / 3} \mathrm{Sr}_{1 / 3} \mathrm{MnO}_{3}$ by using spray and screen printing techniques on various substrates $\left(\mathrm{Al}_{2} \mathrm{O}_{3}\right.$ and $\left.\mathrm{ZrO}_{2}\right)$. The growth conditions are explored in order to optimize the microstructure of the films. The films display a room-temperature magnetoresistance of $0.0012 \% / \mathrm{Oe}$ in the $1 \mathrm{kOe}$ field region. A magnetic sensor is described and tested. (C) 1997 American Institute of Physics. [S0021-8979(97)65308-6]

Due to the existence of a colossal magnetoresistance, in recent years there has been an extraordinary interest in the manganese perovskites $\mathrm{L}_{1-x} \mathrm{~A}_{x} \mathrm{MnO}_{3} \quad(\mathrm{~L}=\mathrm{La}, \mathrm{Pr}, \ldots$; $\mathrm{A}=\mathrm{Ca}, \mathrm{Sr}, \mathrm{Pb}, \ldots) .{ }^{1,2}$ Practical applications of magnetoresistive manganese perovskites, however, have not been yet actively explored. Two obvious reasons are that the maximal magnetoresistive response takes place at the Curie temperature $T_{c}$ and the reported colossal changes of resistivity $\left.[\rho(H=0)-\rho(H)] / \rho(H) \approx 10^{6}\right)$ are only observed when $T_{c}$ is very low (about $70-100 \mathrm{~K}$ ) and under fields of the $10^{4} \mathrm{Oe}$ range.

It is clear that a magnetic sensor should be operated at temperatures close to $T_{c}$ due to the fact that, at this temperature, field sensitivity is higher and thermal stability effects are expected to be weaker. Extensive use in technology will require room-temperature devices and, therefore, due to the fact that the magnetoresistive response becomes weaker when $T_{c}$ increases, the possible applications of these perovskites are challenging. The field sensitivity is also a key issue, as it is expected that sensors should be operated under much lower fields, at most in the kOe range and preferably lower.

On the other hand, materials are most conveniently used in thin-filmlike form, in order to be easily manufacturable, to allow a convenient definition of the sensitive area and, eventually, to tailor appropriately its impedance. Finally, low cost deposition techniques are desirable. To that purpose, thick films offers considerable advantages.

In this paper, we report on the preparation of thick films of $\mathrm{La}_{2 / 3} \mathrm{Sr}_{1 / 3} \mathrm{MnO}_{3}$ by spray and screen printing techniques on various substrates $\left(\mathrm{Al}_{2} \mathrm{O}_{3}\right.$ and $\left.\mathrm{ZrO}_{2}\right)$. At room temperature, resistivity changes up to $1.2 \%$ can be obtained under fields of about $1 \mathrm{kOe}$. We will demonstrate that this sensibility is high enough to fabricate useful magnetic devices that can compete favorably with existing ones, and prototypes of proximity detector devices have been constructed.

Ceramic $\mathrm{La}_{2 / 3} \mathrm{Sr}_{1 / 3} \mathrm{MnO}_{3}$ powders have been prepared by standard solid-state reaction techniques starting from the appropriate mixtures of oxides. A detailed account of the experimental procedure and conditions can be found elsewhere. ${ }^{3}$ Here we will only mention that the maximal firing temperatures for the Sr-based ceramics is $1400{ }^{\circ} \mathrm{C}$ and it is performed under oxygen atmosphere. X-ray diffraction of the as-prepared ceramics shows the presence of the rhombohoedrally distorted perovskite structure.

Ceramic samples have been grounded down to a size of $\leqslant 1 \mu \mathrm{m}$. In order to get the adequate printability, ceramic grains have been suspended in an organic vehicle made by therpineol and ultrasonically dispersed for $15 \mathrm{~min}$. The relative concentration of perovskite to therpineol depends on the method used to make the film. It turns out that for the spray technique the ratio of $70 / 30 \% / w$ leads to the best spraying characteristics of the mixture whereas for the screen printing a $80 / 20 \% / w$ is enough. It will be shown in the following that this difference of concentrations has significant influences on the properties of the films. The Sr-based ceramics have been sprayed or screen printed onto polycrystalline, polished $\mathrm{Al}_{2} \mathrm{O}_{3}(A)$ and $\mathrm{ZrO}_{2}(Z)$ substrates $\left(10 \times 10 \times 1 \mathrm{~mm}^{3}\right)$ at room temperature.

The subsequent thermal treatment involved three steps. In the first one, the films are slowly heated $\left(50^{\circ} \mathrm{C} / \mathrm{h}\right)$ up to a temperature $T_{d}$ and kept at this temperature to allow decomposition of the organic carrier. Two different decomposition temperatures $\left[T_{d}=218{ }^{\circ} \mathrm{C}(10 \mathrm{~min})\right.$, and $\left.250{ }^{\circ} \mathrm{C}(60 \mathrm{~min})\right]$ have been checked. In the second step, the films are heated $\left(300{ }^{\circ} \mathrm{C} / \mathrm{h}\right)$ up to $T_{f}=1400{ }^{\circ} \mathrm{C}$. Finally, the samples are allowed to cool down to room temperature at $300{ }^{\circ} \mathrm{C} / \mathrm{h}$. All steps are performed under flowing $\mathrm{O}_{2}$. Differential scanning calorimetry (DSC) has been used to determine the decomposition temperature of the organic carrier.

Structural characterization of the films has been performed by using a Rigaku x-ray diffractometer and scanning electron microscopy (SEM) inspection. The x-ray diffraction patterns of the grown films can be indexed on the basis of a rhombohedral cell $(R-3 c)$ and it is identical to that of the precursor ceramics. In Fig. 1, we show the pattern corresponding to the $\mathrm{La}_{2 / 3} \mathrm{Sr}_{1 / 3} \mathrm{MnO}_{3}\left(T_{d}=218{ }^{\circ} \mathrm{C}\right)$ film. The refined cell parameters: $a=5.465(5) \AA, \alpha=60.38^{\circ}$ are in good agreement with other literature reports. ${ }^{4}$ No structural differences have been observed for films grown either on $A$ or $Z$ substrates. The SEM images of Fig. 2 show the formation of large grains, larger than $5 \mu \mathrm{m}$, and a rather porous structure with underlying grains well connected. The connectivity results are much better for the films grown on $Z$ than those grown on $A$. The final thickness of the sprayed films is about $10 \mu \mathrm{m}$. In spite of the superior microstructure of the $Z$ films, technical exploit would prefer $A$ films rather than $Z$ films. Consequently, in the following we will report on development of devices based on $A$ films.

Electrical resistivity measurements have been performed on patterned pieces of these films by using de techniques. To 


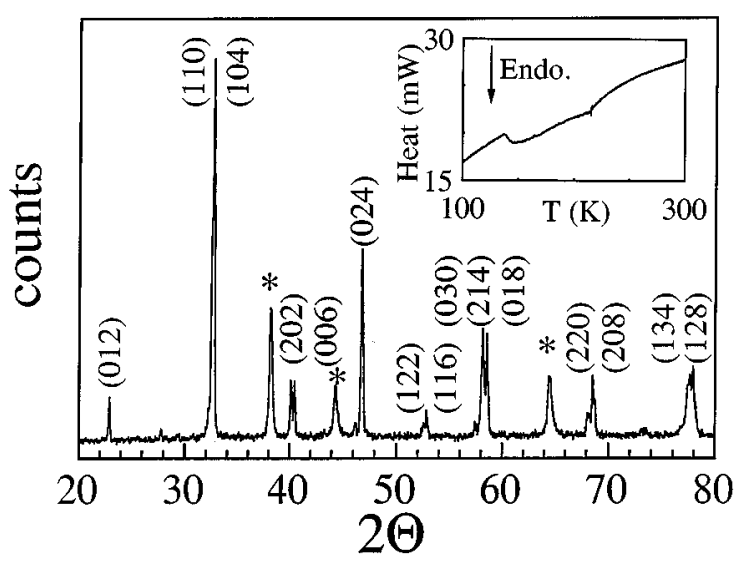

FIG. 1. X-Ray diffraction patterns corresponding to the $\mathrm{La}_{2 / 3} \mathrm{Sr}_{1 / 3} \mathrm{MnO}_{3}$ $\left(T_{f}=1400{ }^{\circ} \mathrm{C}\right)$ film. The substrate holder peaks are marked with asterisks. Inset: The differential scanning calorimetry (DSC) of a perovskite and therpineol $(70 / 30 \% / w)$ mixture.

that purpose, narrow tracks of about $1 \mathrm{~mm}$ wide have been simply patterned by using a scalpel. Contacts have been made by using silver paste. Due to the porosity of the films, absolute values of the resistivity can only be taken as approximate. The field dependence of the resistivity has been measured by using the four-probes method under fields up to $4 \mathrm{kOe}$. This low-field range has been selected because for many applications permanent magnets are going to be used. In Fig. 3 (inset) we show the results obtained at room temperature $(300 \mathrm{~K})$ for the $A$-grown film. The overall variation of resistivity is about $1.2 \%$, that is a magnetoresistance of $0.0012 \% / \mathrm{Oe}$ in the $1 \mathrm{kOe}$ field region. This variation compares with those obtained (at room temperature and under the same field range) on La-Sr-based ceramics and thin films. ${ }^{4}$ Here, it is convenient to recall that for the $\mathrm{Co} / \mathrm{Cu}$ multilayers, the change per unit field is about $0.007 \% / \mathrm{Oe},{ }^{5}$ and in permalloy/Au multilayers values of about $\approx 1 \% / \mathrm{Oe}$ have been found. ${ }^{6}$

The temperature dependence of the resistivity of the $A$ films are shown in Fig. 3. Inspection of these data reveals that the resistivity peak commonly observed at the ferromagnetic ordering temperature occurs at temperatures close to room temperature $\left(T_{c} \approx 280 \mathrm{~K}\right)$ and it is lower for the $A$ film grown by spray than for the $A$ film grown by the screen printing technique. When compared with existing data obtained on bulk samples of the same nominal composition, it turns out that in all cases the resistive peak appears at temperatures significantly lower than those we have measured in the corresponding bulk samples, and which are in the 310$330 \mathrm{~K}$ range. We do believe that these differences arise from a distinct effective doping that can be related to some reduction effect associated to the firing of the organic carrier. To check for this hypothesis, we have grown sprayed films and performed the organic decomposition step at higher temperature. In fact, as shown in the DSC results of Fig. 1 (inset), the therpineol decomposition ends up at about $250{ }^{\circ} \mathrm{C}$. In Fig. 3 we included the $\rho(T)$ data for the $A$ film prepared at $\left(T_{d}=250{ }^{\circ} \mathrm{C}\right)$. It is clear that the maximum in $\rho(T)$ occurs now at higher temperature. Therefore, the differences in $T_{c}$
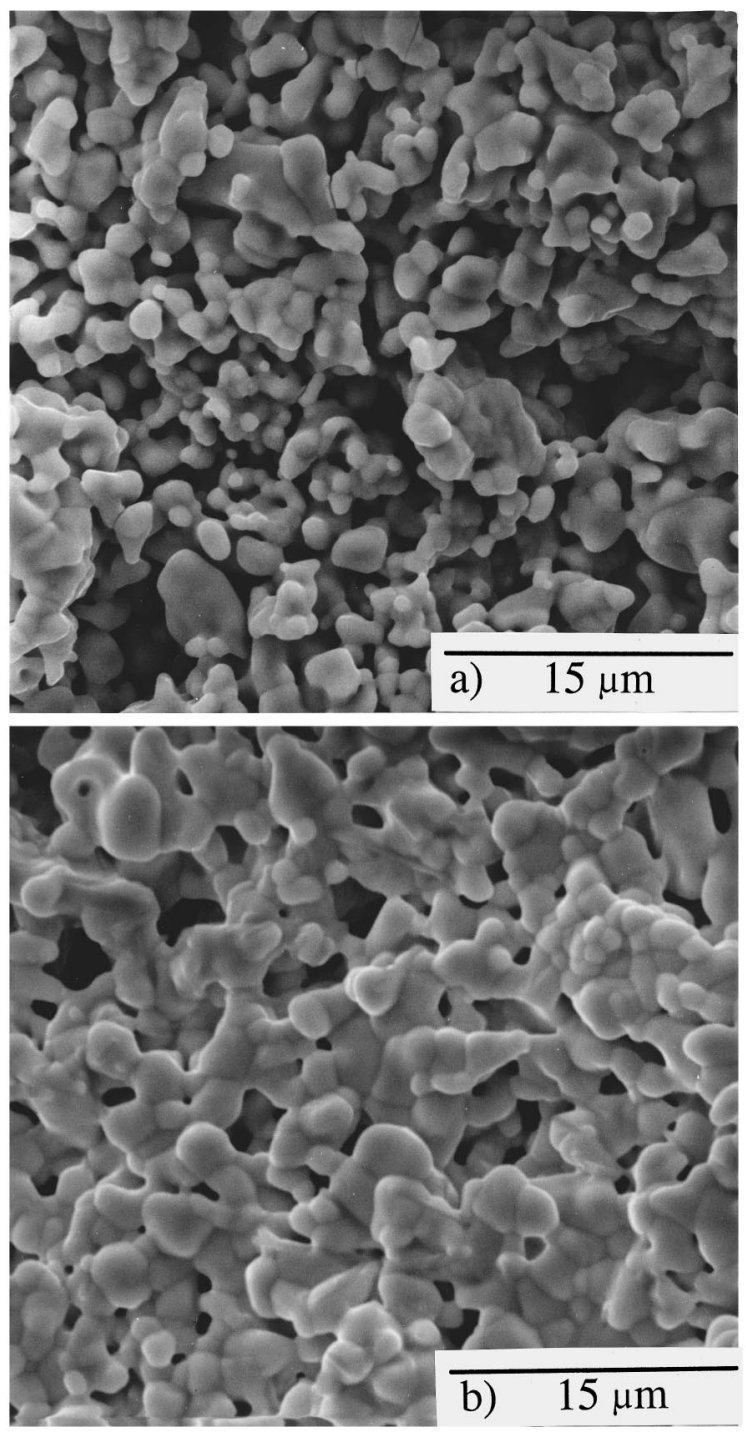

FIG. 2. SEM images $(20 \mathrm{kV})$ of the Sr-based thick films grown on: (a) $\mathrm{Al}_{2} \mathrm{O}_{3}$ and (b) $\mathrm{ZrO}_{2}$ substrates. The marker corresponds to $15 \mu \mathrm{m}$.

among the screen and sprayed films mentioned above can be interpreted as due to the distinct concentration of therpinol used and to the fact that low-temperature decomposition leads to somewhat reducing conditions.

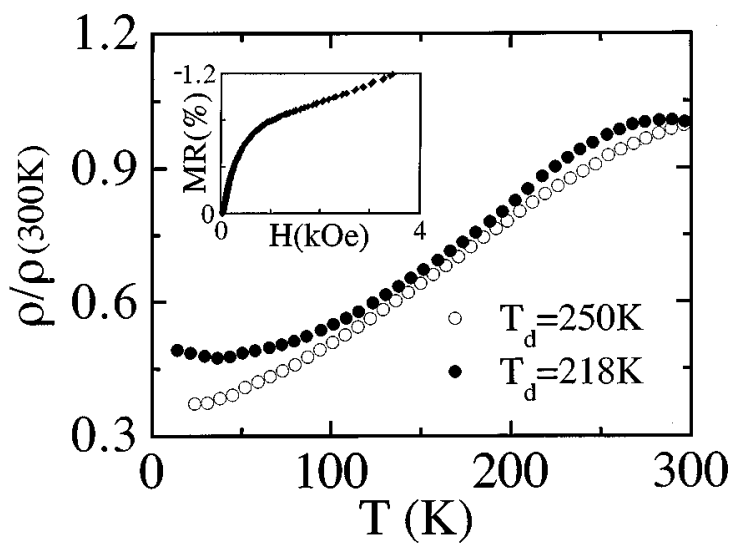

FIG. 3. The temperature dependency of the resistivity of the $\mathrm{Sr}$ films grown $\left(T_{d}=218{ }^{\circ} \mathrm{C}\right.$ and $\left.T_{d}=250{ }^{\circ} \mathrm{C}\right)$ onto $\mathrm{Al}_{2} \mathrm{O}_{3}$ substrates. Inset: roomtemperature field dependence of the magnetoresistance (MR\%). 


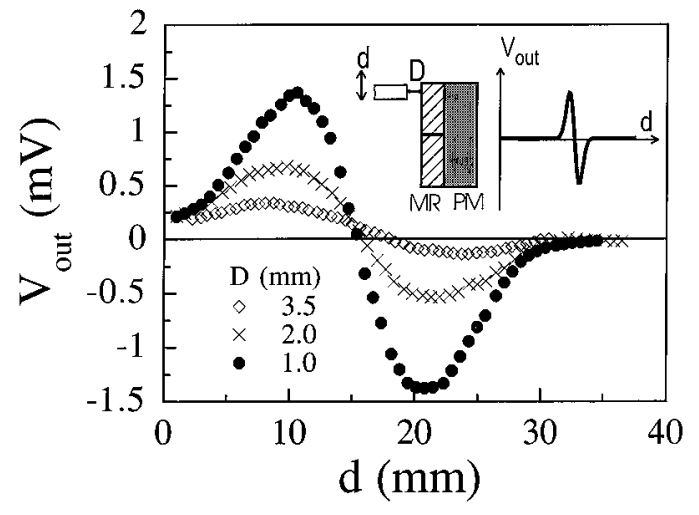

FIG. 4. Output voltage $V_{\text {out }}$ vs distance $(d)$ at various airgaps $(D)$ for a single approaching scalpel. Inset: The sensor's setup and the measuring electrical circuit and the schematic output voltage $V_{\text {out }}$.

A sensor has to be designed to minimize the thermal effects and to optimize the magnetoresistive response. To that purpose, the device is to be operated preferably in one or two active arms of a bridge configuration. The magnetoresistive circuit is formed by tracks patterned on the films; they are series coupled as a voltage divider and the tracks are geometrically adjusted to produce the appropriate resistance in each resistor (see below). The operation point of the device will be fixed by a permanent magnet (ferrite, $H=550$ Oe, $22 \times 48 \times 10 \mathrm{~mm}^{3}$ ) onto which the film is glued. The voltage in the bridge is balanced, in the presence of the permanent magnet, by adjusting the value of external resistors. Figure 4 (inset) illustrates the sensor's setup. In this differential mounting, when a soft iron piece approaches one arm of the sensor, the bridge is unbalanced due to the flux line redistribution over the magnet and each arm of the sensor. Consequently, a finite output voltage $V_{\text {out }}$ is obtained. The expected output wave form is also shown. In our roomtemperature experiments, a scalpel was used as a soft iron piece $\left(10 \times 10 \times 0.3 \mathrm{~mm}^{3}\right)$ and it was displaced by using a computer controlled step motor $(200 \mu \mathrm{m} / \mathrm{step})$ towards the sensor.

Several devices have been constructed and tested. In the case presented here, the sensor is formed by two active arms of a bridge, having sizes of $8 \mathrm{~mm} \times 4 \mathrm{~mm}$ each and a resistance of about $1 \mathrm{k} \Omega$. From the experimental point of view, it is important to notice that in this configuration thermal drifts of the sensors during experiment are to first order canceled. In Fig. 4 we show the typical output voltage $V_{\text {out }}$ measured versus distance at various airgaps $(D)$. As it can be clearly appreciated, the observed response fits nicely to the expected one, and even more the sensitivity is still large enough when the scalpel is moved with an air gap of $D=3.5 \mathrm{~mm}$. Under the present experimental conditions, the lateral resolution of the device at $D=1 \mathrm{~mm}$ is of about $500 \mu \mathrm{V} / \mathrm{mm}$ and in the central part of the sensor the response is linear over about 2 mm length.

Notice that by using this configuration, the device is also sensitive to the direction of motion of the iron piece and, thus, can be used as a digital revolution counter, a linear displacement sensor, a direction dependent contactless switch, angular encoder, etc.

In summary, we have shown that $\mathrm{La}_{2 / 3} \mathrm{Sr}_{1 / 3} \mathrm{MnO}_{3}$ thick films of magnetoresistive manganites can be grown by spray and screen printing techniques onto alumina or zirconia substrates. High-temperature firing of deposited La-Sr-based ceramics leads to films of the appropriate structural and chemical composition that display a significant roomtemperature magnetoresistance. We have demonstrated that even at room temperature the field sensitivity of the resistivity of these manganites is high enough and, thus, offers opportunities for a faster development of magnetic sensors and devices.

Acknowledgments: The authors would like to acknowledge the CICYT-MIDAS (MAT94-1924-CO2), DGICYT (PB92-0849) projects and the Generalitat de Catalunya for financial support.

${ }^{1}$ R. von Helmolt, J. Weckerg, B. Holzapfel, L. Schultz, and K. Samwer, Phys. Rev. Lett. 71, 2331 (1993); S. Jin, T. H. Tiefel, M. McCormack, R. A. Fastnacht, R. Ramesh, and L. H. Chen, Science 264, 413 (1994); S. Jin, H. M. O'Bryan, T. H. Tiefel, M. McCormack, and W. W. Rhodes, Appl. Phys. Lett. 66, 382 (1995).

${ }^{2}$ J. Fontcuberta, B. Martinez, A. Seffar, S. Piñol, J. L. Garcia-Muñoz, and X. Obradors, Phys. Rev. Lett. 76, 1122 (1996); H. Y. Hwang, S.-W. Cheong, P. G. Radaelli, M. Marezio, and B. Batlogg, ibid. 75, 914 (1995); Y. Tomioka, A. Asamitsu, Y. Moritomo, H. Kuwahara, and Y. Tokura, ibid. 74, 5108 (1995).

${ }^{3}$ J. Fontcuberta, B. Martinez, A. Seffar, S. Piñol, J. L. Garcia-Muñoz, and X. Obradors, Europhys. Lett. 34, 379 (1996); J. Fontcuberta, B. Martinez, A. Seffar, S. Piñol, A. Roig, E. Molins, X. Obradors, J. Alonso, and J. M. Gonzales-Calbet, J. Appl. Phys. 79, 5182 (1996).

${ }^{4}$ Y. Tokura, A. Urushibara, Y. Moritomo, T. Arima, A. Asamitsu, G. Kido, and N. Furukawa, J. Phys. Soc. Jpn. 63, 3931 (1994); R. Mahendiran, S. K. Tiwary, A. K. Raychaudhuri, T. V. Ramakrishnan, R. Mahest, R. Ragavittal, and C. N. R. Rao, Phys. Rev. B 53, 3348 (1996).

${ }^{5}$ S. S. P. Parkin, Z. G. Li, and D. J. Smith, Appl. Phys. Lett. 58, 2710 (1991).

${ }^{6}$ S. S. P. Parkin and T. Rabedeau, Appl. Phys. Lett. 68, 1162 (1996). 